CUBO A Mathematical Journal

Vol.15, No 01, (171-189). March 2013

\title{
Discrete Almost Periodic Operators
}

\author{
Alexander Pankov 1 \\ Morgan State University, \\ Department Of Mathematics, \\ 1700 E. Cold Spring Lane, Baltimore, MD 21251, USA. \\ alexander.pankov@morgan.edu
}

\begin{abstract}
This paper deals with discrete almost periodic linear operators in the space of bounded sequences. We study the invertibility of such operators in that space, as well as in the space of almost periodic sequences. One of main results is a discrete version of wellknown First Favard Theorem, and is based on the notion of the envelope of an almost periodic operator. Another result is restricted to finite order operators. It characterizes the invertibility in therms of the operator in question only.
\end{abstract}

\section{RESUMEN}

Este trabajo trata de operadores lineales discretos casi periódicos en el espacio de las secuencias acotadas. Estudiamos la invertibilidad de dichos operadores en ese espacio, así como en el espacio de secuencias casi periódicas. Uno de los resultados principales es una versión discreta del conocido Primer Teorema de Favard, y se basa en la noción de la envolvente de un operador casi periódico. Otro resultado se restringe a los operadores de orden finito. Se caracteriza la invertibilidad solamente en términos del operador en cuestión.

Keywords and Phrases: Almost periodic sequence, discrete operator, Favard condition. 2010 AMS Mathematics Subject Classification: 39A24, 47B39

\footnotetext{
${ }^{1}$ Dedicated to Professor Gaston M. N'Guérékata on the occasion of his 60th Birthday
} 


\section{Introduction}

The theory of almost periodic differential equations has been initiated by J. Favard in his pioneering work [5]. Today the theory is well-developed not only for ordinary differential equations, but also for abstract evolution equations and partial differential equations. Contemporary presentations of the theory can be found in many monographs and survey articles (see, e.g., 1, 3, 1, 9, 11, 13, 14] and references therein).

Difference equations constitute a natural counterpart of the theory of differential equations. We refer to [4 for a contemporary introductory presentation of the theory of difference equations. There is a number of papers that deal with almost periodic difference equations. Most of them concern either special equations (see, e.g. [17]), or first order systems [6, 12. In particular [12] contains certain discrete versions of First and Second Favard Theorems. To the best of our knowledge, there are only few papers dedicated to general almost periodic linear difference equations, or, equivalently, almost periodic discrete linear operators (see [2, 15, 16] and references therein). In particular, in [15 certain discrete version of the First Favard Theorem is obtained (see Corollary 5.2). This version requires coercivity estimate (10) and is not similar to typical Favard type assumptions. Thus, at the moment the theory of almost periodic difference equations is not completely parallel to the theory of almost periodic differential equations.

The aim of the present paper is to fill, at list partially, the gap mentioned above. We accept the functional analytic point of view. This means that we study almost periodic operators in the space $l^{\infty}$ of bounded sequences with values in a finite dimensional space and their restrictions to the space ap of almost periodic sequences. We obtain certain criteria for such an operator $\mathcal{A}$ to have a bounded inverse operator. Equivalently, the invertibility means that the equation $\mathcal{A} x=y$ has a unique solution $x \in l^{\infty}$ for every right hand side $y \in l^{\infty}$. One of our main result, Theorem 5.1, is an exact analogue of the version of First Favard Theorem for differential equations obtained by E. Mukhamadiev in [10] (see also [14]). The second result, Theorem 6.2, is, in a sense , dual to Theorem 5.1, but it holds for operators of finite order. This is an analogue of a result by M. Krasnosel'skii, V. Burd and Y. Kolesov [7.

The paper is organized as follows. Section 2 is a quick reminder of basic facts about almost periodicity. In Section 3 we discuss bounded linear operators in the space $l^{\infty}$. In particular, we introduce important concepts of c-convergence and c-continuity. The main result of the section is Proposition 3.1 which shows that any c-continuous operator is of the form (11) (this is a result by V. Slyusrchuk [16]). Almost periodic operators are introduced in Section 4. Sections 5 and 6 contain our main results.

In what follows, we consider elements of sequence spaces as functions on the set of integers $\mathbb{Z}$. We use the notation [.] to list the values of such a function. On the other hand, the notation $\{\cdot\}$ stands for the lists of elements of a set. 


\section{Almost Periodic Functions and Sequences}

Let $E$ be a Banach space, with the norm $\|\cdot\|_{E}$, over real or complex numbers. We denote by $C_{b}(E)$ the space of bounded continuous functions on $\mathbb{R}$ with values in $E$. This is a Banach space with the norm

$$
\|f\|_{c_{b}}=\sup _{t \in \mathbb{R}}\|f(t)\|_{E} .
$$

A function $f \in C_{b}(E)$ is almost periodic if the family $\{f(\cdot+\tau)\}_{\tau \in \mathbb{R}}$ of shifts is a precompact set in $C_{b}(E)$. Almost periodic functions form a closed subspace $A P(E)$ of $C_{b}(E)$, hence, a Banach space.

By $l^{\infty}(E)$ we denote the space of all bounded two-sided sequences $x=[x(n)]_{n \in \mathbb{Z}}$ with values in $\mathrm{E}$. This is a Banach space endowed with the norm

$$
\|x\|_{l \infty}=\sup _{n \in \mathbb{Z}}\|x(n)\|_{E}
$$

In what follows we also need the space of $E$-valued sequences $l^{1}(E)$, with the norm

$$
\|x\|_{l^{1}}=\sum_{n \in \mathbb{Z}}\|x(n)\|_{E} .
$$

In this paper we do not use other spaces $l^{p}$.

A sequence $x=[x(n)]_{n \in \mathbb{Z}} \in l^{\infty}(E)$ is almost periodic if the set of its $\operatorname{shifts}\{[x(\cdot+q)]\}_{\mathbf{q} \in \mathbb{Z}}$ is a precompact set in $l^{\infty}(E)$. The set of all almost periodic sequences is a closed subspace $a p(E) \subset$ $l^{\infty}(E)$. Hence, $\operatorname{ap}(E)$ is a Banach space.

It is convenient to introduce operators of translation $T_{q}, q \in \mathbb{Z}$, acting in the space $l^{\infty}(E)$ by the formula

$$
\left(T_{q} x\right)(n)=x(n+q), \quad n \in \mathbb{Z} .
$$

These are linear bounded operators. Moreover, they are isometric operators, i.e.,

$$
\left\|\mathrm{T}_{\mathrm{q}} x\right\|_{l^{\infty}}=\|x\|_{l^{\infty}}, \quad \mathrm{q} \in \mathbb{Z} .
$$

The operators $\mathrm{T}_{\mathrm{q}}$ form a one-parameter discrete group of operators, i.e.,

(i) $\mathrm{T}_{\mathrm{q}_{1}+\mathrm{q}_{2}}=\mathrm{T}_{\mathrm{q}_{1}} \mathrm{~T}_{\mathrm{q}_{2}}, \quad \mathrm{q}_{1}, \mathrm{q}_{2} \in \mathbb{Z}$,

(ii) $\mathrm{T}_{0}=\mathrm{I}$,

(iii) $\mathrm{T}_{-\mathrm{q}}=\mathrm{T}_{\mathrm{q}}^{-1}, \quad \mathrm{q} \in \mathbb{Z}$,

where I stands for the identity operator. In terms of these operators the almost periodicity of a sequence $x \in l^{\infty}$ means that the set $\left\{T_{q} x\right\}_{q \in \mathbb{Z}}$ is precompact in $l^{\infty}(E)$.

The following simple statement is well-known (see, e.g., [3, Theorem 1.27]) and clarifies a relation between almost periodic sequences and functions. 
Proposition 2.1. The restriction operator $R: C_{b}(E) \rightarrow l^{\infty}(E)$ defined by $f \mapsto[f(n)]_{n \in \mathbb{Z}}$ maps $\mathrm{AP}(\mathrm{E})$ onto ap $(\mathrm{E})$. Furthermore, there exists a linear operator $\mathrm{J}: \mathrm{E} \rightarrow \mathrm{C}_{\mathrm{b}}(\mathrm{E})$, an extension operator, such that $(\mathrm{J} x)(\mathrm{n})=\chi(\mathrm{n})$ for all $\mathrm{n} \in \mathbb{Z},\|J x\|_{\mathrm{C}_{\mathrm{b}}}=\|x\|_{l_{\infty}}$ for all $\mathrm{x} \in \mathrm{l}^{\infty}(\mathrm{E})$, and $\mathrm{J}(\operatorname{ap}(\mathrm{E})) \subset$ $A P(E)$.

Making use of Proposition 2.1, one can transfer many results about almost periodic functions to almost periodic sequences.

Finally, we introduce a simple, but important, notion of periodization. Given a positive integer j, let

$$
Q_{j}=\{n \in \mathbb{Z} \mid j-1 \leq n \leq j\} .
$$

For any $x \in l^{\infty}(E)$, its $2 j$-periodization is a $2 j$-periodic sequence, say $x_{j}=\left[x_{j}(n)\right]_{n \in \mathbb{Z}}$, such that $x_{j}(n)=x(n)$ for all $n \in Q_{j}$. Obviously, $x_{j} \stackrel{c}{\rightarrow} x$ and, hence, the space $\operatorname{ap}(E)$ is c-dense in $l^{\infty}(E)$.

\section{Linear Operators in $l^{\infty}$}

In the rest of the paper $\mathrm{E}$ stands for a finite dimensional Banach space. For sequences in the space $l^{\infty}(E)$ one can introduces several kinds of convergence. In this paper we use the standard convergence with respect to the norm of $l^{\infty}(E)$ and the so-called c-convergence. A sequence $x_{k} \in l^{\infty}(E)$ c-converges to $x \in l^{\infty}(E)$ (in symbols $x_{k} \stackrel{c}{\rightarrow} x$ ) if the sequence $x_{k}$ is bounded in $l^{\infty}(E)$ and $x_{k}(n) \rightarrow x(n)$ for all $n \in \mathbb{Z}$. In this case we also write $x=c$ - $\lim x_{k}$.

By $L\left(l^{\infty}(E)\right)$ we denote the Banach algebra of all bounded linear operators in $l^{\infty}(E)$. An operator $\mathcal{A} \in \mathrm{L}\left(l^{\infty}(\mathrm{E})\right)$ is c-continuous if for any sequence $x_{\mathrm{k}} \in l^{\infty}(\mathrm{E})$ such that $x_{\mathrm{k}} \stackrel{\mathrm{c}}{\rightarrow} \mathrm{x}$ we have that $\mathcal{A x} \mathrm{x}_{\mathrm{k}} \stackrel{\mathrm{c}}{\rightarrow} \mathcal{A x}$. The set of all c-continuous operators is a closed subalgebra of the Banach algebra $\mathrm{L}\left(l^{\infty}(\mathrm{E})\right)$ (see [2, Proposition 1]). We denote this subalgebra by $\mathrm{L}_{\mathrm{c}}\left(l^{\infty}(\mathrm{E})\right)$.

We consider operators of the form

$$
(\mathcal{A x})(\mathrm{n})=\sum_{m \in \mathbb{Z}} A(n, m) x(m), \quad n \in \mathbb{Z}
$$

where $A(n, m) \in L(E)$. The double sequence $A=[A(n, m)]_{n, m \in \mathbb{Z}}$ is called the kernel of $\mathcal{A}$. Given such a kernel $A$, we set

$$
\|A\|=\sup _{n \in \mathbb{Z}} \sum_{m \in \mathbb{Z}}\|A(n, m)\|_{L(E)} .
$$

An alternative representation of operator (1) is

$$
(\mathcal{A} x)(n)=\sum_{k \in \mathbb{Z}} a(n, k) x(n+k), \quad n \in \mathbb{Z}
$$

where $a(n, k)=A(n, n+k)$ are called the coefficients of $\mathcal{A}$. It is easily seen that, for double

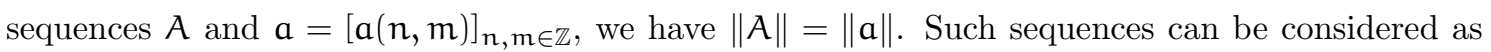


sequences indexed by $n$ with values in the space of sequences indexed by $m$. From this point of view the norm defined by (2) is exactly the $l^{\infty}\left(l^{1}(\mathrm{~L}(\mathrm{E}))\right)$-norm.

The nontrivial part of the next result goes back to [16. Since this paper is not available in English, we preset the proof here.

Proposition 3.1. A linear operator $\mathcal{A}$ in $l^{\infty}(\mathrm{E})$ is a bounded $\mathrm{c}$-continuous operator if and only if $\mathcal{A}$ is of the form (1) with $\|\mathrm{A}\|<\infty$. Moreover, in this case

$$
\|\mathcal{A}\|_{\mathrm{L}\left(l^{\infty}(\mathrm{E})\right)}=\|\mathrm{A}\| .
$$

Proof. Suppose that $\|A\|<\infty$. Then it is well-known, and easily seen, that

$$
\|\mathcal{A}\|_{\mathrm{L}\left(l^{\infty}(\mathrm{E})\right)} \leq\|\mathrm{A}\|
$$

Hence, $\mathcal{A} \in \mathrm{L}\left(l^{\infty}(\mathrm{E})\right)$.

To prove that $\mathcal{A}$ is $\mathrm{c}$-continuous, suppose that $\mathrm{x}_{\mathrm{k}} \stackrel{\mathrm{c}}{\rightarrow} 0$. Obviously, the sequence $\mathcal{A} \mathrm{x}_{\mathrm{k}}$ is bounded in $l^{\infty}(E)$, and we need to show that $\left(\mathcal{A} x_{k}\right)(n) \rightarrow 0$ in $E$ for every $n \in \mathbb{Z}$. For any positive $\mathrm{N} \in \mathbb{Z}$

$$
\left\|\left(\mathcal{A} x_{k}\right)(n)\right\|_{E} \leq\left(\sum_{|m| \leq N}+\sum_{|m|>N}\right)\|A(n, m)\|_{L(E)}\left\|x_{k}(m)\right\|_{E} .
$$

Since the sequence $x_{k}$ is bounded in $l^{\infty}(E)$ and $\|A\|<\infty$, choosing $N$ large enough we can make the second sum in the right-hand side sufficiently small. Next, since $x_{k} \stackrel{c}{\rightarrow} 0$, the first sum is sufficiently small provided $k$ is large enough. This proves the first statement.

Suppose that $\mathcal{A} \in \mathrm{L}_{\mathrm{c}}\left(\mathrm{l}^{\infty}(\mathrm{E})\right)$. First, we define its kernel as follows. Denote by $\mathrm{J}_{\mathrm{m}}: \mathrm{E} \rightarrow l^{\infty}(\mathrm{E})$, $\mathrm{m} \in \mathbb{Z}$, the operator defined by

$$
\left(J_{m} \mathfrak{u}\right)(\mathfrak{n})= \begin{cases}u & \text { if } n=m \\ 0 & \text { otherwise }\end{cases}
$$

The operator $P_{n}: l^{\infty}(E) \rightarrow E, n \in \mathbb{Z}$, is defined by $P_{n} x=x(n)$. For all $n, m \in \mathbb{Z}$, we set

$$
\mathrm{A}(\mathrm{n}, \mathrm{m})=\mathrm{P}_{\mathrm{n}} \mathcal{A} \mathrm{J}_{\mathrm{m}} \text {. }
$$

Obviously, $A(n, m) \in L(E)$.

Now we prove (11), where the right-hand side converges in $l^{\infty}(E)$ for each $n \in \mathbb{Z}$. Indeed, given $x \in l^{\infty}(E)$, we set

$$
x_{k}(n)=\left\{\begin{array}{cl}
x(n) & \text { if }|n| \leq k \\
0 & \text { otherwise }
\end{array}\right.
$$

It is easily seen that $\mathrm{x}_{\mathrm{k}} \stackrel{\mathrm{c}}{\rightarrow} \mathrm{x}$. Since $\mathcal{A}$ is c-continuous, then for every $\mathrm{n} \in \mathbb{Z}$

$$
\left(\mathcal{A} x_{k}\right)(n)=\sum_{|m| \leq k} A(n, m) x(m) \rightarrow(\mathcal{A} x)(n)
$$


as required.

Let us prove that

$$
\|A\| \leq\|\mathcal{A}\|_{\mathrm{L}\left(l^{\infty}(\mathrm{E})\right)}
$$

For each $k \in \mathbb{Z}$, we consider an element $x_{k} \in l^{\infty}(E)$ such that $\left\|x_{k}(n)\right\|_{E}=1$ and

$$
\left\|A(k, n) x_{k}(n)\right\|_{E}=\|A(k, n)\|_{L(E)}
$$

for all $n \in \mathbb{Z}$. Since $\operatorname{dim} E<\infty$, such a sequence exists. Then

$$
\sum_{m \in \mathbb{Z}}\|A(k, m)\|_{L(E)}=\left\|\left(\mathcal{A} x_{k}\right)(k)\right\|_{E} \leq\left\|\mathcal{A} x_{k}\right\|_{l^{\infty}} \leq\|\mathcal{A}\|_{\mathrm{L}\left(l^{\infty}\right)}
$$

because $\left\|x_{k}\right\|_{l^{\infty}(E)}=1$. This implies the required.

Proposition 3.1 and [2, Proposition 3] imply immediately

Corollary 3.2. Suppose that $\mathcal{A} \in \mathrm{L}_{\mathrm{c}}\left(\mathrm{l}^{\infty}(\mathrm{E})\right)$ has a bounded inverse operator. Then the inverse operator is c-continuous and, hence, is of the form

$$
\left(\mathcal{A}^{-1} x\right)(\mathrm{n})=\sum_{\mathrm{m} \in \mathbb{Z}} \mathrm{G}(\mathrm{n}, \mathrm{m}) x(\mathrm{n}), \quad \mathrm{n} \in \mathbb{Z}
$$

with $\|\mathrm{G}\|=\left\|\mathcal{A}^{-1}\right\|_{\mathrm{L}\left(l^{\infty}(\mathrm{E})\right)}<\infty$.

The kernel G in Corollary 3.2 is often called the Green function of the operator $\mathcal{A}^{-1}$.

Remark 3.3. Under the assumption of Corollary 3.2, suppose in addition that the kernel A satisfies

$$
\|A(m, n)\|_{L(E)} \leq \frac{c}{(1+|n-m|)^{\alpha}}, \quad m, n \in \mathbb{Z}
$$

with $\mathrm{c}>0$ and $\alpha>2$. Then for every $\theta>0$ small enough there exists $\mathrm{c}_{\theta}>0$ such that the Green function satisfies

$$
\|\mathrm{G}(\mathrm{m}, \mathrm{n})\|_{\mathrm{L}(\mathrm{E})} \leq \frac{\mathrm{c}_{\theta}}{(1+|\mathrm{n}-\mathrm{m}|)^{\alpha-1-\theta}}, \quad \mathrm{m}, \mathrm{n} \in \mathbb{Z} .
$$

Furthermore, if

$$
\|A(m, n)\|_{L(E)} \leq c \exp (-\delta|n-m|), \quad m, n \in \mathbb{Z},
$$

with $\mathrm{c}>0$ and $\delta>0$, then there exist $\mathrm{c}_{1}>0$ and $\varepsilon>0$ such that

$$
\|G(m, n)\|_{L(E)} \leq c_{1} \exp (-\varepsilon|n-m|), \quad m, n \in \mathbb{Z},
$$

(see [2] and [14]). 


\section{Almost Periodic Operators}

We say that $\mathcal{A} \in \mathrm{L}\left(l^{\infty}(\mathrm{E})\right)$ is an almost periodic operator if the sequence of operators $\left[\mathrm{T}_{\mathrm{q}} \mathcal{A} \mathrm{T}_{-\mathrm{q}}\right]_{\mathrm{q} \in \mathbb{Z}}$ is an almost periodic sequence with values in $\mathrm{L}\left(l^{\infty}(\mathrm{E})\right)$.

Proposition 4.1. An operator $\mathcal{A} \in \mathrm{L}\left(l^{\infty}(\mathrm{E})\right)$ is almost periodic if and only if the $\operatorname{set}\left\{\mathrm{T}_{\mathrm{q}} \mathcal{A} \mathrm{T}_{-\mathrm{q}}\right\}_{\mathrm{q} \in \mathbb{Z}}$ is precompact in $\mathrm{L}\left(l^{\infty}(\mathrm{E})\right)$.

For the proof we refer to [2, Proposition 6].

The envelope, or hull, $\mathrm{H}(\mathcal{A})$ of an almost periodic operator $\mathcal{A} \in \mathrm{L}\left(l^{\infty}(\mathrm{E})\right)$ is the closure of the set $\left\{\mathrm{T}_{\mathrm{q}} \mathcal{A} \mathrm{T}_{-\mathrm{q}}\right\}_{\mathrm{q} \in \mathbb{Z}}$ in the space $\mathrm{L}\left(\mathrm{l}^{\infty}(\mathrm{E})\right)$. This is a compact set.

Now we collect some properties of almost periodic operators obtained in 2 .

Proposition 4.2. Suppose that $\mathcal{A} \in \mathrm{L}\left(1^{\infty}(\mathrm{E})\right)$ is almost periodic operator. Then the following statements hold:

(a) $\mathcal{A}(\mathrm{ap}(\mathrm{E})) \subset \mathrm{ap}(\mathrm{E})$.

(b) If $\mathcal{A}$ has a bounded inverse operator, then $\mathcal{A}^{-1}$ is an almost periodic operator and, hence, $\mathcal{A}_{\mid \mathrm{ap}(\mathrm{E})}$ has a bounded inverse operator in $\mathrm{L}(\mathrm{ap}(\mathrm{E}))$. Moreover, all operators in the envelope $\mathrm{H}(\mathcal{A})$ are invertible and

$$
\mathrm{H}\left(\mathcal{A}^{-1}\right)=\left\{\tilde{\mathcal{A}}^{-1}: \tilde{\mathcal{A}} \in \mathrm{H}(\mathcal{A})\right\} .
$$

(c) If, in addition, $\mathcal{A}$ is of the form (1), the kernel $\mathrm{A}$ satisfies (4) with $\mathrm{c}>0$ and $\alpha>2$, and if $\mathcal{A}_{\mathrm{ap}(\mathrm{E})}$ has a bounded inverse operator in $\mathrm{L}(\mathrm{ap}(\mathrm{E}))$, then the operator $\mathcal{A}$ has a bounded inverse operator in $\mathrm{L}\left(l^{\infty}(\mathrm{E})\right)$.

Remark 4.3. Actually, under the assumptions of Proposition 4.2(c) the operator $\mathcal{A}$ is $\mathbf{c}$-continuous.

Proposition 4.4. Suppose that $\mathcal{A} \in \mathrm{L}\left(\mathrm{l}^{\infty}(\mathrm{E})\right)$ is a c-continuous operator of the form (1)). The following statements are equivalent:

(i) The operator $\mathcal{A}$ is almost periodic.

(ii) The kernel $\mathrm{A}$ is almost periodic along the diagonal with respect to norm (2), i.e., the sequence of kernels $[A(\cdot+q, \cdot+q)]_{\mathbf{q} \in \mathbb{Z}}$ is an almost periodic sequence in with values in the space of kernels endowed with the norm $\|\cdot\|$.

(iii) The sequence $[\mathrm{a}(\mathrm{n}, \cdot)]_{\mathrm{n} \in \mathbb{Z}}$ of coefficients is an almost periodic sequence with values in $\mathrm{l}^{1}(\mathrm{~L}(\mathrm{E}))$.

Proof. A straightforward verification shows that $[A(n+q, m+q)]$ is the kernel of the operator $\mathrm{T}_{\mathrm{q}} \mathcal{A} \mathrm{T}_{-\mathrm{q}}$. Now the required follows immediately from Proposition 3.1 and remarks after equation (3). 
Remark 4.5. If an almost periodic operator $\mathcal{A} \in \mathrm{L}\left(1^{\infty}(\mathrm{E})\right)$ is of the form (1), then every operator $\tilde{\mathcal{A}} \in \mathrm{H}(\mathcal{A})$ is of the same form. The kernel of $\tilde{\mathcal{A}}$ is a limit point of the $\operatorname{set}\{\mathrm{A}(\cdot+\mathrm{q}, \cdot+\mathrm{q})\}_{\mathrm{q} \in \mathbb{Z}}$ in the space of kernels with respect to the norm $\|\cdot\|$, while the collection of the coefficients is the limit point of the set $\{\mathrm{a}(\cdot+\mathrm{q}, \cdot)\}_{\mathbf{q} \in \mathbb{Z}}$ in the space $\mathrm{l}^{1}(\mathrm{~L}(\mathrm{E}))$.

\section{$5 \quad$ Favard Type Theorem}

In this section we prove the following result of Favard type for almost periodic operators in $l^{\infty}(E)$.

Theorem 5.1. An almost periodic operator $\mathcal{A} \in \mathrm{L}_{\mathrm{c}}\left(l^{\infty}(\mathrm{E})\right)$ has a bounded inverse operator if and only if the following condition is satisfied:

(F) every operator in the envelope $\mathrm{H}(\mathcal{A})$ is injective.

Proof. If $\mathcal{A} \in \mathrm{L}_{\mathrm{c}}\left(l^{\infty}(\mathrm{E})\right)$ is invertible, then, by Proposition $4.2(\mathrm{~b})$, all operators in the envelope are invertible, and (F) follows.

Now suppose that (F) is satisfied. To prove that the operator $\mathcal{A}$ has a bounded inverse, it is enough to show that for any $y \in l^{\infty}(E)$ there exists $x \in l^{\infty}(E)$ such that

$$
\mathcal{A x}=\mathrm{y} .
$$

For any positive integer $j$, denote by $x_{j}=\left[x_{j}(n)\right]_{n \in \mathbb{Z}}$ the $2 j$-periodization of $x$. Similarly, we denote by $a_{j}=\left[a_{j}(n, k)\right]_{n, k \in \mathbb{Z}}$ the $2 j$-periodization of $a=[a(n, k)]_{n, k \in \mathbb{Z}}$ with respect to the variable $n$. According to equation (3), $a_{j}$ generates an operator $\mathcal{A}_{j}$ that belongs to $L_{c}(E)$. Notice that $2 \mathrm{j}$-periodic sequences form a finite dimensional subspace of $l^{\infty}(\mathrm{E})$, and $\mathcal{A}_{j}$ leaves that subspace invariant.

We solve the equation

$$
\mathcal{A}_{j} x_{j}=y_{j}
$$

in the subspace of $2 j$-periodic sequences provided $j$ is large enough. Since the problem is finite dimensional, it is sufficient to show that the associated homogeneous problem has only zero solution. Assuming the contrary, one can find a sequence $j_{l} \rightarrow \infty$ such that $\mathcal{A}_{j_{l}} x_{j_{l}}=0$ for some $x_{j_{l}} \in l^{\infty}(E)$ with $\left\|x_{j_{l}}\right\|_{l \infty}=1$. Then there exists $q_{l} \in Q_{j_{l}}$ such that $\left\|x_{j_{l}}\left(q_{l}\right)\right\|_{E}=1$. We put $\tilde{x}_{l}=T_{q_{l}} x_{j_{l}}$ and $\tilde{\mathcal{A}}_{l}=\mathrm{T}_{\mathrm{q}_{l}} \mathcal{A}_{\mathrm{j}_{l}} \mathrm{~T}_{-\mathrm{q}_{l}}$. Then $\left\|\tilde{\mathrm{x}}_{\mathrm{l}}\right\|_{l^{\infty}}=1,\left\|\tilde{\mathrm{x}}_{l}(0)\right\|_{\mathrm{E}}=1$ and $\tilde{\mathcal{A}}_{l} \tilde{\mathrm{x}}_{l}=0$. Passing to a subsequence, we can assume that $\tilde{x}_{l} \stackrel{c}{\rightarrow} \tilde{x}$ and

$$
\mathcal{A}_{\mathrm{l}}=\mathrm{T}_{\mathrm{q}} \mathcal{A} \mathrm{T}_{-\mathrm{q}_{\mathrm{l}}} \rightarrow \tilde{\mathcal{A}}
$$

in $\mathrm{L}\left(l^{\infty}(\mathrm{E})\right)$. Obviously, $\|\tilde{\mathrm{x}}(0)\|_{\mathrm{E}}=1$ and $\tilde{\mathcal{A}} \in \mathrm{H}(\mathcal{A})$. Given $n \in \mathbb{Z}$ we have

$$
(\tilde{\mathcal{A}} \tilde{x})(n)=\left[(\tilde{\mathcal{A}} \tilde{x})(n)-\left(\tilde{\mathcal{A}} \tilde{x}_{l}\right)(n)\right]+\left[\left(\tilde{\mathcal{A}} \tilde{x}_{l}\right)(n)-\left(\mathcal{A}_{l} \tilde{x}_{l}\right)(n)\right]+\left(\mathcal{A}_{l} \tilde{x}_{l}\right)(n) .
$$


Here the first term in the right-hand side tends to 0 since the operator $\tilde{\mathcal{A}}$ is c-continuous,

$$
\left\|\left(\tilde{\mathcal{A}} \tilde{x}_{l}\right)(\mathrm{n})-\left(\mathcal{A}_{l} \tilde{x}_{l}\right)(\mathrm{n})\right\|_{\mathrm{E}} \leq\left\|\tilde{\mathcal{A}}-\mathcal{A}_{l}\right\|_{\mathrm{L}\left(l^{\infty}(\mathrm{E})\right)} \rightarrow 0,
$$

and

$$
\left.\left(\mathcal{A}_{l} \tilde{x}_{l}\right)(\mathrm{n})=\left[\left(\mathrm{T}_{\mathrm{q}_{l}} \mathcal{A} \mathrm{T}_{-\mathrm{q}_{\mathrm{l}}}\right) \mathrm{T}_{\mathrm{q}_{\mathrm{l}}} \mathrm{x}_{\mathrm{l}}\right)\right](\mathrm{n})=\left(\mathrm{T}_{\mathrm{q}_{l}} \mathcal{A} \mathrm{x}_{\mathrm{j}_{\mathrm{l}}}\right)(\mathrm{n})=\left(\mathrm{T}_{\mathrm{q}_{l}} \mathcal{A}_{\mathrm{j}_{l}} \mathrm{x}_{\mathrm{j}_{l}}\right)(\mathrm{n})=0
$$

provided $\left|q_{l}\right| \geq|\mathfrak{n}|$. Hence, $\tilde{\mathcal{A}} \tilde{x}=0$, which contradicts to condition $(F)$. Thus, equation (9) has a unique $2 j$-periodic solution $x_{j}$ for all sufficiently large $j$.

The sequence $x_{j}$ is bounded in the space $l^{\infty}(E)$. For if this is not so, we can find a subsequence $x_{j_{l}}$ such that $\left\|x_{j_{l}}\right\|_{l^{\infty}} \rightarrow \infty$. Set

$$
z_{l}=x_{j_{l}} /\left\|x_{j_{l}}\right\|_{l \infty} .
$$

Then

$$
\mathcal{A}_{l_{l}} z_{l}=y_{j_{l}} /\left\|x_{j_{l}}\right\|_{l \infty} .
$$

Arguing exactly as above, we obtain that there is a nonzero $x \in l^{\infty}(E)$ such that $\tilde{\mathcal{A}} x=0$, and we arrive at contradiction to condition $(\mathrm{F})$.

Now since the sequence $x_{j}$ is bounded in $l^{\infty}(E)$, then $x_{j} \stackrel{c}{\rightarrow} x$ along a subsequence. It is easy to see that $\mathcal{A x}=\mathrm{y}$.

The following result is obtained in [15].

Corollary 5.2. Let $\mathcal{A} \in \mathrm{L}_{\mathfrak{c}}\left(l^{\infty}(\mathrm{E})\right)$ be an almost periodic operator. If there exists a constant $c_{0}>0$ such that

$$
\|\mathcal{A} x\|_{1_{\infty}} \geq \mathrm{c}_{0}\|x\|_{l^{\infty}}
$$

for all $x \in l^{\infty}(\mathrm{E})$, then the operator $\mathcal{A}$ is invertible in $l^{\infty}(\mathrm{E})$.

Proof. Since the operators $\mathrm{T}_{\mathrm{q}}$ are isometric, then, by the definition of the envelope, all operators in $\mathrm{E}(\mathcal{A})$ satisfy inequality (10). This implies condition $(\mathrm{F})$, and we conclude.

By Proposition 4.2(b), we obtain the following

Corollary 5.3. If an almost periodic operator $\mathcal{A} \in \mathrm{L}_{\mathfrak{c}}\left(l^{\infty}(\mathrm{E})\right)$ satisfies condition $(\mathrm{F})$, then the operator $\mathcal{A}_{\mid \mathrm{ap}(\mathrm{E})}$ has a bounded inverse operator in the space ap $(\mathrm{E})$.

Furthermore, Proposition 4.2(c) implies

Corollary 5.4. Suppose that the kernel $\mathrm{A}$ of an almost periodic operator $\mathcal{A} \in \mathrm{L}_{\mathfrak{c}}\left(l^{\infty}(\mathrm{E})\right)$ satisfies satisfies inequality (4) with $\mathrm{c}>0$ and $\alpha>2$. Then the following statements are equivalent:

(i) The operator $\mathcal{A}$ satisfies condition $(\mathrm{F})$; 
(ii) The operator $\mathcal{A}$ has a bounded inverse operator in the space $\mathrm{l}^{\infty}(\mathrm{E})$;

(iii) The operator $\mathcal{A}_{\mid \mathrm{ap}(\mathrm{E})}$ has a bounded inverse operator in the space ap(E).

\section{Operators of Finite Order}

Now, under certain additional assumptions, we obtain yet another criterion for an almost periodic operator to be invertible. This result is, in a sense, dual to Theorem 5.1

In this section we consider operators of finite order. These are of the form

$$
(\mathcal{A} x)(n)=\sum_{k=k_{1}}^{k_{2}} a(n, k) x(n+k), \quad n \in \mathbb{Z},
$$

where $a\left(n, k_{1}\right)$ and $a\left(n, k_{2}\right)$ are non-zero operators in E. The number $k_{2}-k_{1}$ is called the order of $\mathcal{A}$. In what follows we always suppose that the order of $\mathcal{A}$ is greater than zero. The kernel $[\mathrm{A}(\mathrm{n}, \mathrm{m})]$ of $\mathcal{A}$ vanishes outside the strip $\left\{(\mathrm{n}, \mathrm{m}): \mathrm{k}_{1} \leq \mathrm{m}-\mathrm{n} \leq \mathrm{k}_{2}\right\}$.

We impose the following assumptions:

(A1) $\sup \left\{\|a(n, k)\|_{L(E)}: n \in \mathbb{Z}, k_{1} \leq k \leq k_{2}\right\}<\infty$;

(A2) For all $\mathrm{n} \in \mathbb{Z}$ the operators $\mathrm{a}\left(\mathrm{n}, \mathrm{k}_{1}\right)$ and $\mathrm{a}\left(\mathrm{n}, \mathrm{k}_{2}\right)$ are invertible in $\mathrm{L}(\mathrm{E})$, and there exists a constant $\mathrm{C}>0$ independent of $\mathrm{n}$ such that

$$
\left\|a^{-1}\left(n, k_{1}\right)\right\|_{L(E)} \leq C
$$

and

$$
\left\|a^{-1}\left(n, k_{2}\right)\right\|_{L(E)} \leq C
$$

Assumption (A1) is necessary and sufficient for an operator $\mathcal{A}$ of the form (11) to be a bounded linear operator in $l^{\infty}(\mathrm{E})$. In this case, $\mathcal{A}$ is c-continuous automatically. Assumption (A2) is natural because it is necessary for the existence of bounded inverse operator $\mathcal{A}^{-1}$. Let us also mention that the operator $\mathcal{A}$ is almost periodic if and only if for any $k, k_{1} \leq k \leq k_{2}$, the sequence $[a(n, k)]_{n \in \mathbb{Z}}$ is almost periodic. Furthermore, the envelope $\mathrm{H}(\mathcal{A})$ of any almost periodic operator of finite order consists of operators of finite order.

The following simple, but important, property is well-known.

Proposition 6.1. Assume that an operator $\mathcal{A}$ of finite order $\geq 1$ satisfies (A1) and (A2). Then its null space

$$
\left\{x \in l^{\infty}(\mathrm{E}) \mid \mathcal{A} x=0\right\}
$$

is finite dimensional. 
Proof. Let $\mathrm{d} \geq 1$ be the order of $\mathcal{A}$. Assumption (A2) implies immediately that the linear mapping from the null space into the space $E^{\mathrm{d}}$ defined by

$$
x=[x(n)]_{\mathfrak{n} \in \mathbb{Z}} \mapsto\left(x\left(k_{1}\right), x\left(k_{1}+1\right), \ldots, x\left(k_{2}-1\right)\right)
$$

is one-to-one.

The main result of the section is the following.

Theorem 6.2. Suppose that an operator $\mathcal{A}$ of the form (11) is almost periodic, of order $\mathrm{d} \geq 1$, and satisfies assumptions (A1) and (A2). Then the following statements are equivalent:

(i) The range of operator $\mathcal{A}$ contains ap (E);

(ii) The operator $\mathcal{A}$ has an inverse operator in $\mathrm{L}\left(l^{\infty}(\mathrm{E})\right)$;

(iii) The operator $\mathcal{A}_{\mid \mathrm{ap}(\mathrm{E})}$ has an inverse operator in $\mathrm{L}(\mathrm{ap}(\mathrm{E}))$.

Proof. The equivalence of (ii) and (iii) follows from Proposition 4.2, Obviously, (ii) implies (i).

Now we prove that $(\mathfrak{i})$ implies (ii). Assuming (i), we have to show that the equation

$$
\mathcal{A x}=\mathrm{y}
$$

has a unique solution $x \in l^{\infty}(E)$ for any $y \in l^{\infty}(E)$.

Claim 1. For any $\mathrm{y} \in \mathrm{ap}(\mathrm{E})$ there exists a solution $\mathrm{x} \in \mathrm{ap}(\mathrm{E})$ of equation (12) such that

$$
\|x\|_{l^{\infty}} \leq \mathrm{C}\|\mathrm{y}\|_{l_{\infty}},
$$

with some constant $\mathrm{C}>0$ independent of $\mathrm{y}$.

Denote by $\mathrm{V}$ the preimage of $a p(U)$ under the operator $\mathcal{A}$. Since ap $(\mathrm{E})$ is a closed subspace of $l^{\infty}(\mathrm{E})$ and $\mathcal{A}$ is a bounded operator, $V$ is a closed subspace as well, hence, a Banach space. The operator $\mathcal{A}_{\mid V}$ maps $V$ onto ap $(\mathrm{E})$. Now the required is a particular case of a well-known result about linear operators from a Banach space onto a Banach space (for an excellent presentation see [8]).

Claim 2. For any $\mathrm{y} \in \mathrm{l}^{\infty}(\mathrm{E})$ there exists a solution $\mathrm{x} \in \mathrm{l}^{\infty}(\mathrm{E})$ of equation (12) that satisfies estimate (13).

Let $y_{j}$ be the $2 j$-periodization of $y$. Obviously, $\left\|y_{j}\right\|_{l_{\infty}} \leq\|y\|_{l_{\infty}}$. By Claim 1 , there exists an almost periodic solution $x_{j}$ of equation (12), with $y$ replaced by $y_{j}$, and

$$
\left\|x_{j}\right\|_{l^{\infty}} \leq \mathrm{C}\left\|y_{j}\right\|_{l^{\infty}} \leq \mathrm{C}\|y\|_{l^{\infty}} .
$$


Hence, along a subsequence, $x_{j_{k}} \stackrel{c}{\rightarrow} x$, and $x$ is a solution of (12) that satisfies (13).

Claim 3. Each operator $\tilde{\mathcal{A}} \in \mathrm{H}(\mathcal{A})$ maps $l^{\infty}(\mathrm{E})$ onto $l^{\infty}(\mathrm{E})$.

Let

$$
\tilde{\mathcal{A}}=\lim \mathrm{T}_{\mathrm{q}_{j}} \mathcal{A} \mathrm{T}_{-\mathrm{q}_{j}} \in \mathrm{H}(\mathcal{A}) \text {. }
$$

By Claim 2, given $y \in l^{\infty}(E)$ there exists $x_{j} \in l^{\infty}(E)$ such that $\mathcal{A} x_{j}=T_{-q_{j}} y$ and

$$
\left\|x_{j}\right\|_{l \infty} \leq C\left\|T_{-q_{j}} y\right\|_{l \infty}=C\|y\|_{l \infty} .
$$

Setting $\tilde{x}_{j}=T_{q_{j}} x_{j}$, we have that

$$
\mathrm{T}_{\mathrm{q}_{j}} \mathcal{A} \mathrm{T}_{-\mathrm{q}_{j}} \tilde{\mathrm{x}}_{\mathrm{j}}=\mathrm{y}
$$

Passing to a subsequence, we can suppose that there exists $x \in l^{\infty}$ such that $\tilde{x}_{j} \stackrel{c}{\rightarrow} x$. Passing to the limit in equation (14), we see that $\tilde{\mathcal{A}} x=y$.

Claim 4. If $\mathrm{y} \in \mathrm{ap}(\mathrm{E})$, then every solution $\mathrm{x} \in \mathrm{l}^{\infty}(\mathrm{E})$ of equation (12) is almost periodic.

Due to Claim 1, it is enough to show that every solution of the homogeneous equation, i.e., with $y=0$, is almost periodic. Assume the contrary. Then there exists $x \in l^{\infty}$ such that $\mathcal{A x}=0$ and $x$ is not almost periodic, i.e., the family of shifts $\left\{T_{q} x\right\}_{q \in \mathbb{Z}}$ is not precompact. Then there exist $\varepsilon_{0}>0$ and an infinite set of integers $\left\{\mathbf{q}_{\mathbf{j}}\right\}$ such that

$$
\left\|x_{j}-x_{i}\right\|_{l_{\infty}} \geq \varepsilon_{0}
$$

for $i \neq j$, where $x_{j}=T_{q_{j}} x$. Without loss of generality, we can suppose that

$$
\mathrm{T}_{\mathrm{q}_{j}} \mathcal{A} \mathrm{T}_{-\mathrm{q}_{j}} \rightarrow \tilde{\mathcal{A}} \in \mathrm{H}(\mathcal{A})
$$

in the space $L\left(l^{\infty}(E)\right)$. Since

$$
\left\|\tilde{\mathcal{A}} x_{j}\right\|_{l^{\infty}}=\left\|\left(\tilde{\mathcal{A}}-\mathrm{T}_{\mathrm{q}_{j}} \mathcal{A} \mathrm{T}_{-\mathrm{q}_{j}}\right) \mathrm{x}_{\mathrm{j}}\right\|_{l^{\infty}} \leq\left\|\tilde{\mathcal{A}}-\mathrm{T}_{\mathrm{q}_{j}} \mathcal{A} \mathrm{T}_{-\mathrm{q}_{j}}\right\|_{\mathrm{L}\left(l^{\infty}(\mathrm{E})\right)}\|x\|_{l^{\infty}},
$$

then

$$
\tilde{\mathcal{A}} x_{j} \rightarrow 0
$$

in $l^{\infty}(E)$ as $j \rightarrow \infty$.

Let $\tilde{V}_{0}$ be the null space of the operator $\tilde{\mathcal{A}}$. By Proposition 6.1 this is a finite dimensional subspace in $l^{\infty}(E)$. Hence, there exists a bounded projector $\tilde{P}_{0}$ in $l^{\infty}(E)$ onto $\tilde{V}_{0}$. Set $\tilde{P}_{1}=I-\tilde{P}_{0}$ and $\tilde{V}_{1}=\tilde{P}_{1}\left(l^{\infty}(E)\right)$. Obviously, $\tilde{\mathcal{A}} \tilde{P}_{0} x_{j}=0$. Hence, by (16),

$$
\tilde{\mathcal{A}} \tilde{\mathrm{P}}_{1} x_{\mathrm{j}} \rightarrow 0
$$

in $l^{\infty}(E)$ as $j \rightarrow \infty$. 
The restriction $\tilde{\mathcal{A}}_{\mid \tilde{V}_{1}}$ is one-to-one and, by Claim 3, maps $\tilde{V}_{1}$ onto $l^{\infty}(E)$. Hence, by (17),

$$
\tilde{\mathrm{P}}_{1} x_{j} \rightarrow 0
$$

in $l^{\infty}(E)$ as $j \rightarrow \infty$. By the triangle inequality,

$$
\left\|\tilde{P}_{0} x_{j}-\tilde{P}_{0} x_{i}\right\|_{l_{\infty}} \geq\left\|x_{j}-x_{i}\right\|_{l_{\infty}}-\left\|\tilde{P}_{1} x_{j}\right\|_{l_{\infty}}-\left\|\tilde{P}_{1} x_{i}\right\|_{l_{\infty}} .
$$

Now (15) and (18) imply that for any $\varepsilon_{1} \in\left(0, \varepsilon_{0}\right)$

$$
\left\|\tilde{P}_{0} x_{j}-\tilde{P}_{0} x_{i}\right\|_{l^{\infty}} \geq \varepsilon_{1},
$$

whenever both $\boldsymbol{j}$ and $i$ are large enough. Hence, the set $\left\{\tilde{P}_{0} x_{j}\right\}$ is not a precompact set.

On the other side, $\left\{\tilde{P}_{0} x_{j}\right\}$ is a bounded subset of a finite dimensional space $\tilde{V}_{0}$. Hence, it is precompact, and we arrive at a contradiction.

Claim 5. The null space $\mathrm{V}_{0}$ of the operator $\mathcal{A}$ is trivial.

Without loss of generality, we can assume that $k_{2}=1$. The restriction operator $R: l^{\infty}(E) \rightarrow$ $E^{d}$ is defined by

$$
R: x=[x(n)]_{\mathfrak{n} \in \mathbb{Z}} \mapsto\left(x\left(k_{1}\right), x\left(k_{1}+1\right), \ldots, x(0)\right) .
$$

As we have mentioned in the proof of Proposition 6.1, $R$ maps $V_{0}$ into $E^{d}$ in one-to-one manner. We set $V^{0}=R\left(V_{0}\right)$. This is a linear subspace of $E^{d}$. Choose any direct complement $V^{1}$ to $V^{0}$ in $\mathrm{E}^{\mathrm{d}}$ and set

$$
\mathrm{V}_{1}=\left\{x \in l^{\infty}(\mathrm{E}): \mathrm{Rx} \in \mathrm{V}^{1}\right\} .
$$

Then $V_{0} \oplus V_{1}=l^{\infty}(E)$ and $\mathcal{A}\left(V_{1}\right)=l^{\infty}(E)$. The operator

$$
\mathcal{A}_{\mid \mathrm{V}_{1}}: \mathrm{V}_{1} \rightarrow l^{\infty}(\mathrm{E})
$$

is one-to-one and onto and, hence, has a bounded inverse denoted by

$$
B: l^{\infty}(E) \rightarrow V_{1} .
$$

Now we prove that $V_{0}=\{0\}$. Suppose that $\mathcal{A x}=0$, and $x \neq 0$. By Claim $4, x$ is an almost periodic sequence. Let $\theta_{j}=\left[\theta_{j}(n)\right]_{\mathfrak{n} \in \mathbb{Z}}$ be a (scalar valued) sequence defined by

$$
\theta_{j}(n)=\left\{\begin{array}{lll}
0 & \text { if } & n \leq 0 \\
n & \text { if } & 1 \leq n \leq j \\
j & \text { if } & n>j
\end{array}\right.
$$

Consider the sequence

$$
x_{j}=\theta_{j} \cdot x=\left[\theta_{j}(n) x(n)\right]_{n \in \mathbb{Z}} .
$$


Obviously, $x_{j} \in l^{\infty}(E)$. Moreover, $x_{j} \in V_{1}$ because $x_{j}(n)=0$ if $n \leq 0$.

We have

$$
\mathcal{A} x_{j}=\theta_{j} \cdot \mathcal{A} x+z_{j}=z_{j},
$$

where $z_{\mathfrak{j}}=\left[z_{\mathfrak{j}}(\mathrm{n})\right]_{\mathrm{n} \in \mathbb{Z}}$ with

$$
z_{j}(n)=\sum_{k=k_{1}}^{1}\left(\theta_{j}(n+k)-\theta_{j}(n)\right) a(n, k) x(n+k), \quad n \in \mathbb{Z} .
$$

It is easily seen that

$$
\left|\theta_{j}(n+k)-\theta_{j}(n)\right| \leq d
$$

for all $n \in \mathbb{Z}$ and integer $k \in\left[k_{1}, 1\right]$. Hence, $\left\|z_{j}\right\|_{l_{\infty}}$ is bounded above by a constant independent of $j$. Since $x_{j} \in V_{1}$, we have that $x_{j}=B z_{j}$ and, hence, $\left\|x_{j}\right\|_{l_{\infty}} \leq C$, where $C>0$ is independent on j. In particular,

$$
j\|x(j)\|_{E} \leq\left\|x_{j}\right\|_{l^{\infty}} \leq C .
$$

Hence, $x(j) \rightarrow 0$ as $j \rightarrow \infty$, which implies that $x=0$ because, by Claim $3, x$ is almost periodic.

This completes the proof of the theorem.

Combining Theorem 6.2 and Corollary [5.4, we obtain

Corollary 6.3. Under the assumptions of Theorem 6.2 the following statements are equivalent:

(i) The operator $\mathcal{A}$ satisfies condition $(\mathrm{F})$ of Theorem [5.1;

(ii) The operator $\mathcal{A}$ has a bounded inverse in $\mathrm{L}\left(\mathrm{l}^{\infty}(\mathrm{E})\right)$;

(iii) The operator $\mathcal{A}$ maps $l^{\infty}(\mathrm{E})$ onto $l^{\infty}(\mathrm{E})$;

(iv) The restriction $\mathcal{A}_{\mid \mathrm{ap}(\mathrm{E})}$ has a bounded inverse in $\mathrm{L}(\mathrm{ap}(\mathrm{E}))$;

(v) The restriction $\mathcal{A}_{\mid \mathrm{ap}(\mathrm{E})}$ maps ap (E) onto ap(E).

\section{Received: November 2012. Revised: December 2012.}

\section{References}

[1] L. Amerio, G. Prouse, Almost Periodic Functions and Functional Equations, Van Nostrand, New York, 1971.

[2] G. Bruno, A. Pankov, Y. Tverdokhleb, On almost periodic operators in the spaces of sequences, Acta Applicandae Mathematicae, 65 (2001), 153-167. 
[3] C. Corduneanu, Almost Periodic Functions, Chelsea Publ., New York, 1989.

[4] S. Elaydi, An Introduction to Difference Equations, Springer, New York, 2005.

[5] J. Favard, Sur les équations différentielles linéaires à coefficients presque-périodiques, Acta Math, 51 (1927), 31-81.

[6] A. Halanay, D. Wexler, Teoria Calitativă a Sistemelor cu Impulsuri (Romanian), Ed. Acad. Rep. Soc. Rom., Bucharest, 1968.

[7] M. A. Krasnosel'skii, V. Sh. Burd, Yu. S. Kolesov, Nonlinear Almost Periodic Oscillations, Wiley, New York, 1973 (English Edition).

[8] S. G.Krein, Linear Equations in Banach Spaces, Birkhäuser, Boston, 1972 (English Edition).

[9] B. M. Levitan, V. V. Zhikov, Almost Periodic Functions and Differential Equations, Cambridge Univ. Press, Campbridge, 1982 (English Edition).

[10] E. Mukhamadiev, On the invertibility of elliptic partial differential operators (Russian), Dokl. Akad. Nauk SSSR, 205 (1972), 1292-1295; English translation: Soviet Math. Dokl., 13 (1972), $1122-1126$.

[11] G. M. N'Guérékata, Almost Automorphic and Almost Periodic Functions in Abstract Spaces, Kluwer, New York, 2001.

[12] A. Pankov, On the Favard theory for impulse evolution equations (Russian), Rev. Roumaine Math. Pures Appl.,25 (1980), 385-401.

[13] A. Pankov, Bounded and Almost Periodic Solutions of Nonlinear Operator Differential Equations, Kluwer, Dordrecht, 1990 (English Edition).

[14] M. A. Shubin, Almost periodic functions and differential equations, Uspekhi Mat. Nauk, 33 no 2 (1978), 3-47; English translation:Russian Math. Surv., 33 no 2 (1978), 1-52.

[15] V. E. Slyusarchuk, Invertibility of almost periodic operators generated by discrete systems (Russian), Ukr. Mat. Zhurn., 31 (1979), 460-463.

[16] V. E. Slyusarchuk, On representation of bounded solutions of discrete linear systems (Russian), Ukr. Mat. Zhurn., 39 (1987), 210-215.

[17] S. Zhang, P. Liu, K. Gopalsamy, Almost periodic solutions of nonautonomous linear difference equations, Applicable Analysis, 81 (2002), 281-301. 